Int. J. Dev. Biol. 60: 71-76 (2016)

doi: $10.1387 / \mathrm{ijdb} .150365 \mathrm{jb}$

\title{
Matrix Gla Protein expression pattern in the early avian embryo
}

\author{
ELIZABETH CORREIA ${ }^{1,2}$, NATÉRCIA CONCEIÇÃO ${ }^{1,3}$, M. LEONOR CANCELA ${ }^{* 1,3}$ and JOSÉ A. BELO ${ }^{* 1,2,4}$
}

${ }^{1}$ Department of Biomedical Sciences and Medicine, University of Algarve, Faro, ${ }^{2}$ Center for Biomedical Research (CBMR), Universidade do Algarve, Campus de Gambelas, Faro, ${ }^{3}$ Centre of Marine Sciences, University of Algarve, ${ }^{4}$ CEDOC, NOVA Medical School / Faculdade de Ciências Médicas, Universidade Nova de Lisboa, Lisboa, Portugal

\begin{abstract}
MGP (Matrix Gla Protein) is an extracellular matrix vitamin K dependent protein previously identified as a physiological inhibitor of calcification and shown to be well conserved among vertebrates during evolution. MGP is involved in other mechanisms such as TGF- $\beta$ and BMP activity, and a proposed modulator of cell-matrix interactions. MGP is expressed early in vertebrate development although its role has not been clarified. Previous work in the chicken embryo found MGP localization predominantly in the aorta and aortic valve base, but no data is available earlier in development. Here we examined MGP expression pattern using whole-mount in situ hybridization and histological sectioning during the initial stages of chick development. MGP was first detected at HH10 in the head and in the forming dorsal aorta. At the moment of the onset of blood circulation, MGP was expressed additionally in the venous plexus which will remodel into the vitelline arteries. By E2.25, it is clear that the vitelline arteries are MGP positive. MGP expression progresses centrifugally throughout the area vasculosa of the yolk sac. Between stages HH17 and HH19 MGP is seen in the dorsal aorta, heart, notochord, nephric duct, roof plate, vitelline arteries and in the yolk sac, beneath main arterial branches and in the vicinity of several vessels and venules. MGP expression persists in these areas at least until E4.5. These data suggest that MGP expression could be associated with cell migration and differentiation and to the onset of angiogenesis in the developing chick embryo. This data has biomedical relevance by pointing to the potential use of chick embryo explants to study molecules involved in artery calcification.
\end{abstract}

KEY WORDS: MGP, angiogenesis, heart development, brain development, yolk sac

Matrix Gla Protein (MGP) is a small secreted protein present in the extracellular matrix (ECM) that is known to be a physiological inhibitor of calcification. The correlation between the absence of functional MGP and aberrant calcification of both arteries and cartilage was clearly established following the development of the MGP knock-out mice (Luo et al., 1997). In humans, MGP mutations are the genetic basis of the Keutel syndrome (KS), a rare autosomal recessive disease characterized by abnormal cartilage calcification, brachytelephalangism, peripheral pulmonary artery stenosis, hearing loss, and facial abnormalities (Keutel etal., 1971; Hur et al., 2005).

Multiple functions have been associated to MGP. These functions include the regulation of calcium phosphate transport (Price et al., 2002), the activity of TGF- $\beta 1$ (Bostrom et al., 2004), the inhibition of BMP2 (Wallin et al., 2000), BMP4, and BMP7 by direct binding
(Yao et al., 2011), the maintenance of BMP and Notch signaling balance and the maintenance of normal endothelial cell function (Sharma and Albig, 2013; Yao et al., 2013).

Nonetheless, MGP appears early in development, being expressed in a specific pattern in embryonic tissues, suggesting a developmentally regulated expression which could be associated to cell differentiation and proliferation (Cancela et al., 2014). In the avian embryo its expression was reported at E 14 in the aorta and at the base of the avian aortic valve predominantly in proteoglycan-rich spongiosa region and on the fibrosa surface of the valve (Alfieri et al., 2010).

Despite a lower homology of chick (Gene ID: 395912) and

Abbreviations used in this paper: ECM, extracellular matrix; MGP, matrix gla protein.

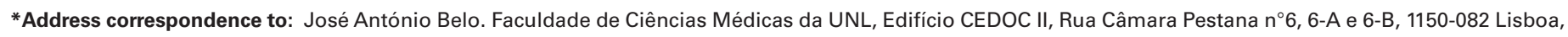

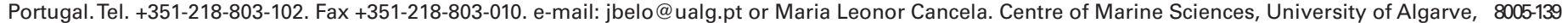
Faro, Portugal. Tel. +351-289.-800971. Fax +351-289-818353. e-mail: Icancela@ualg.pt
}

Accepted: 23 December 2015

ISSN: Online 1696-3547, Print 0214-6282 
mammalian MGP when compared to homology among mammalian MGPs (Wiedemann 1998), they possess identical protein domains (signal peptide; phosphorylation domain (SxxSxxS); $\gamma$-glutamyl carboxylase recognition site; Gla domain) and there is a conservation of all residues thought to be critical for function such as the Gla residues, the ANxF proteolytic cleavage site, the cysteines required for disulphyde bridge and the $\mathrm{C}$ terminal arginine (RR) cleavage site (Cancela et al., 2014). In addition, MGP has been shown to be involved in chondrocyte cell differentiation during limb development of chick embryo (Yagami et al., 1999). The purpose of this study was to investigate the sites of MGP mRNA expression in the initial stages of chick development for which no information is currently available, in order to access the potential use of chick embryo explant cultures to test the activity of novel drugs/molecules with anti-artery calcification potential.

\section{Results}

MGPmRNA transcripts were first seen as two longitudinal parallel stripes, equally distant from the notochord, near the somites at

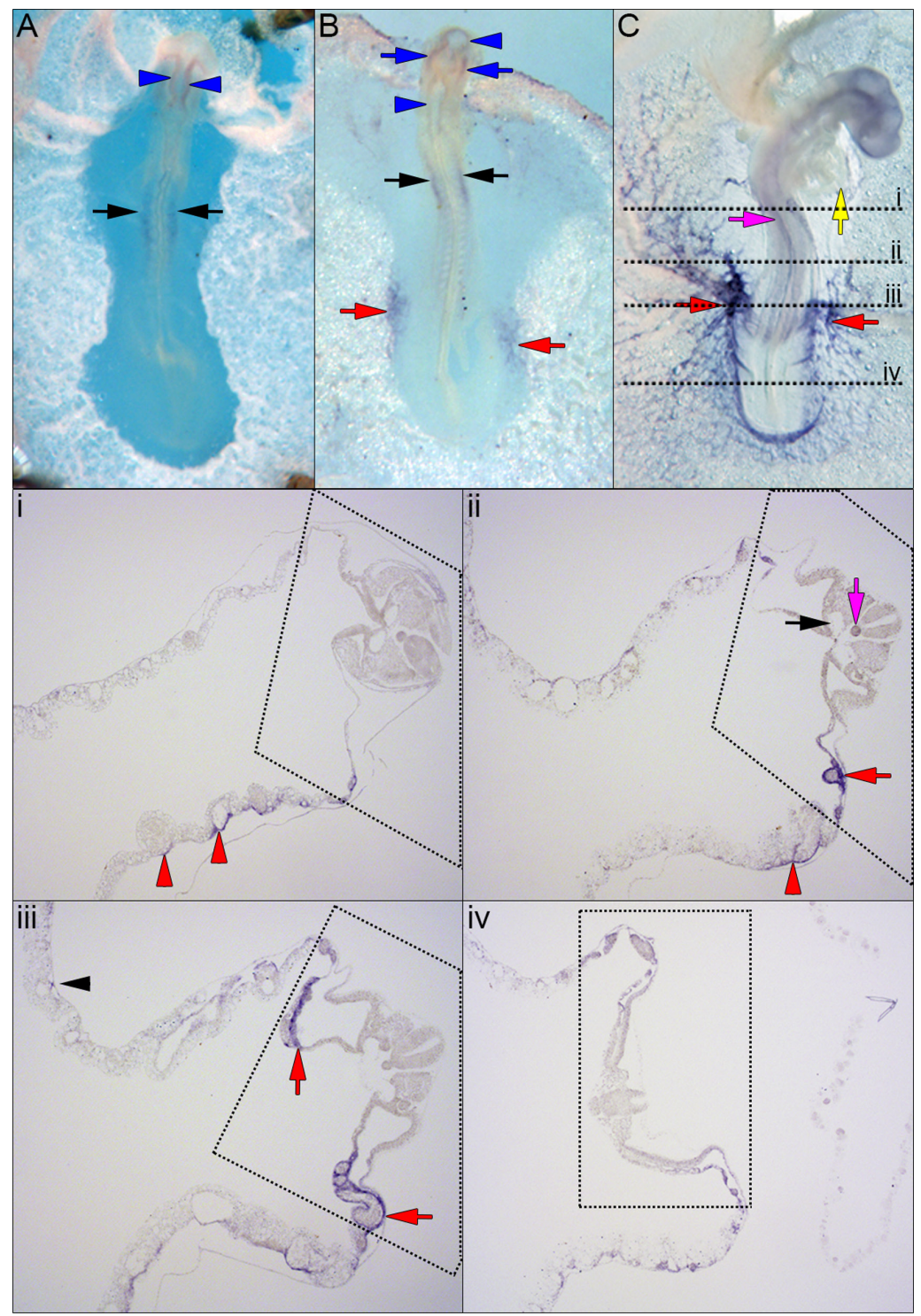

$\mathrm{HH} 10+$ (Hamburger-Hamilton staging; Fig. 1A, black arrows). At $\mathrm{HH} 13$ it remains on the two stripes beside the first seven pairs of somites (Fig. 1B-black arrows). By HH15 (Fig. 1C) no staining is visible in the dorsal aorta, however it is present in some portions of the notochord (Fig. 1ii-black arrow and pink arrow, respectively). At $\mathrm{HH} 18$ MGP transcripts are seen in the dorsal aorta and notochord, but also in the vicinity of the internal carotids, which are the anterior extension of the dorsal aorta. This expression is present in the surrounding areas of the internal carotids facing the core of the embryo (Fig. 3Da-De). Further in development (HH21-23; Fig. 3Cablack arrow), the dorsal aorta remains positive for MGP transcripts. The notochord also maintains MGP expression, however now the expression seems to transcend its limits (Fig. 3Ca-pink arrows). By HH25 MGP expression in the dorsal aorta and notochord was no longer observed (data not shown).

$M G P$ is expressed on a plexus of small caliber sized vessels (18ss; Fig. 1B, red arrows) that constitute the future vitelline arteries (le Noble et al., 2004). In Fig. 1C (red arrows; HH15), the newly formed vitelline arteries can be seen expressing MGP transcripts. This intense expression occurs evenly in the wall of the lateral vitelline arteries (red arrows in Fig. 1ii and Fig. 1iii), in the area pellucida. As soon as (or shortly after) the vitelline artery branches enter the area vasculosa of the area opaca, a change on the expression of MGP can be observed, becoming then restricted to the dorsal part of these vessels (Fig. $1 \mathrm{i}$-iii, red arrowheads and Fig. 2B, red arrows and red arrowheads).

As embryonic day 2 advances, MGPexpression spreads through the area vasculosa. By HH18 MGP expression can be seen covering the full-extension of the area opaca, reaching up to the sinus terminalis. MGP transcripts were not detected neither in the sinus terminalis nor in the vitelline veins (Fig. 2B, red hollow arrows) at any developmental stage analyzed. MGP expression in the area vasculosa not only appears in the dorsal part of vitelline artery branches but also in the vicinity of several vessels and venules, as well as in what seems to be small vessels beneath main arterial branches (Fig. $3 \mathrm{Aa}-\mathrm{Bc}$ ). At this developmental

Fig. 1. MGP expression during pre-circulatory and early circulatory stages. WISH using DIG labelled MGP (A-C) was performed on embryos from $\mathrm{HH} 10+$ to 15. (A) At HH10+, MGP is expressed as two stripes alongside the first 7 pairs of somites (black arrows) and in the mesencephalon (blue arrowheads). (B) MGP expression, at $\mathrm{HH}_{13}$, is present in the prosencephalon and rhombencephalon (blue arrowheads), in two stripes bilateral to the mesencephalon (blue arrows), on a plexus of small caliber sized vessels (red arrows) that constitute the future vitelline arteries, and it also remains on the two stripes beside the first 7 pairs of somites (black arrows). (C) By HH15, MGP expression is seen in some portions of the notochord (pink arrow; ii), with great intensity in the early vitelline arteries (red arrows). This expression is observed uniformly throughout the lateral wall of the vitelline arteries (ii-iii; red arrows), at least in the area pellucida. The expression in the area opaca seems to be restricted to the dorsal part of these vessels (i-ii; red arrowheads). No expression was detected in the heart (C; yellow arrow), anterior vitelline vein (C; hollow red arrow) or dorsal aorta (ii; black arrow). Embryo images (A-C) from a dorsal view. (i-iv) transverse sections of embryo in (C), with dorsal side displayed to the right. Dashed lines delimitate the area pellucida. 


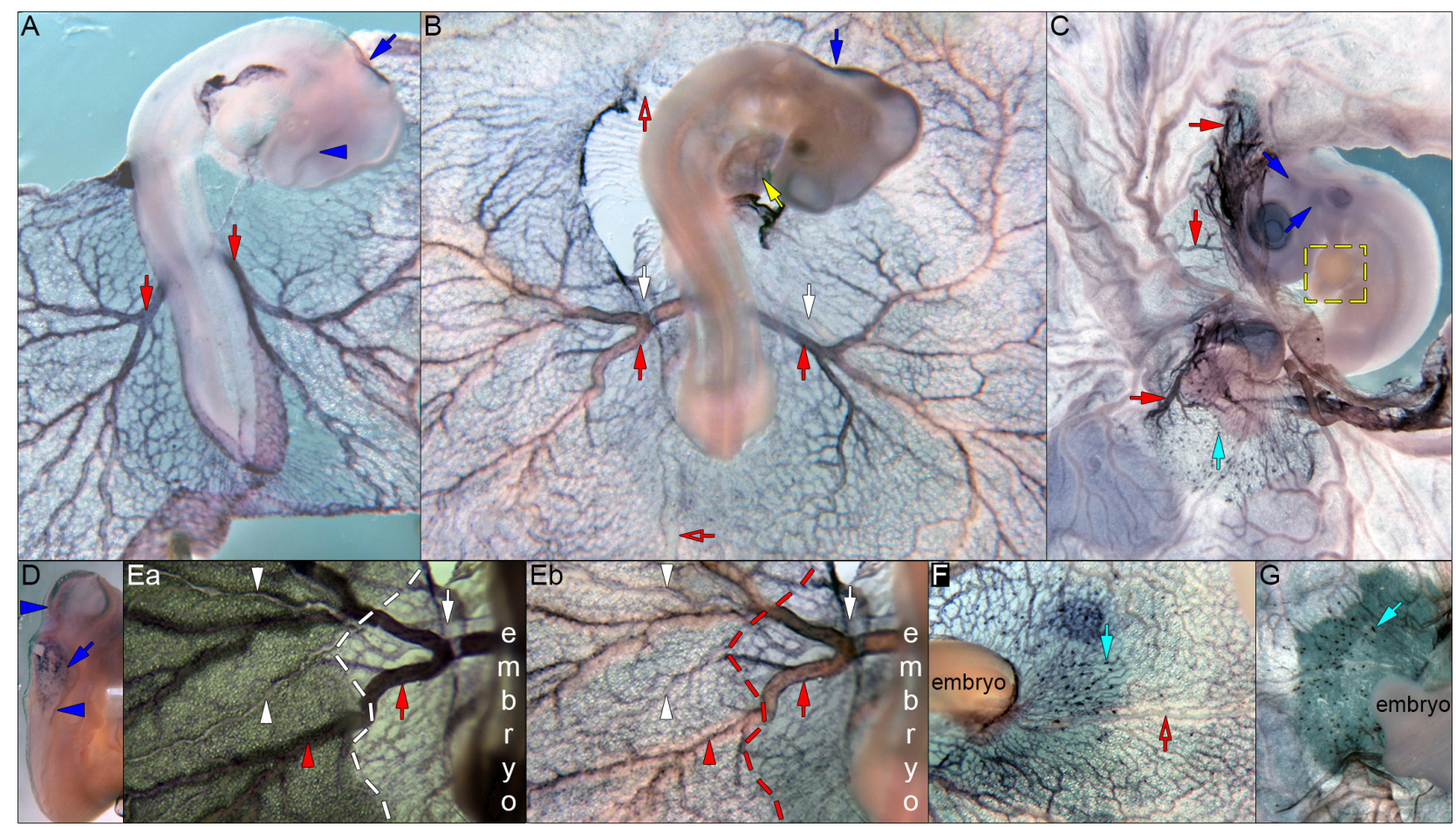

Fig. 2. MGP expression during yolk sac vasculature remodeling. WISH using DIG labelled MGP (A-G) was performed on embryos from HH17 to HH21. (A) At HH17, MGP is expressed in the vitelline arteries (red arrows) and throughout the yolk sac vasculature, in the roof plate (blue arrow) and in the forming diencephalon (blue arrowheads). (B) MGP expression, at HH18, remains in the roof plate (blue arrow), in the vitelline arteries (red arrows) and throughout the yolk sac vasculature. Additionally it appears in the heart (yellow arrow). MGP expression is absent from the anterior vitelline vein (red hollow arrow) and from the newly formed posterior vitelline vein (red hollow arrow) and lateral vitelline veins (white arrows). (C) By HH21, MGP is observed predominantly in the vasculature of the area pellucida (red arrows), excluding the embryo. Still, in the area pellucida, blood islands can be observed (cyan arrow). Although, staining is not visible in the roof plate at this developmental stage, same regions appear positive for MGP in the head (blue arrows). The expression in the heart is maintained (yellow dashed boxed detail). (D) Detail of an HH18 roof plate. (E a,b) Detail of the left side of the embryo in (B), respectively in dark field view and bright field view. Dashed lines delimitate the area pellucida. White arrows, lateral vitelline veins in the area pellucida; white arrowheads, lateral vitelline vein branches in the area opaca; red arrows, vitelline arteries in the area pellucida; red arrowheads, vitelline artery branches in the area opaca. (F,G) Detail of blood islands (cyan arrows) and posterior vitelline vein (red hollow arrow) of embryos at HH19 and $H H 21$, respectively. All images show embryos in a dorsal view. A, anterior; P, posterior.

stage, there is a partial overlap of MGP and Ephrin-B2 expression (Fig. 3Ha-Id). Both genes seem to be expressed in the dorsal aorta, in the vitelline arteries and in the yolk sac. In Fig. $3 \mathrm{Ha}$, an even expression of Ephrin-B2 can be seen (purple staining, blue arrow) in the endothelium of the artery. On the other hand, MGP expression is seen only in the dorsal part of this vessel (red staining, red arrows, Fig. $3 \mathrm{Ha}$ and $\mathrm{Hb}$ ). MGP expression level in the embryo is very subtle but very intense in the vasculature of the yolk sac. Interestingly, at this same developmental stage, Ephrin-B2 expression levels are the complete opposite, being more intense in the embryo. MGP was also observed at different developmental stages (in-between E2.5 - 3 and E3 - 3.5) in the posterior part of the embryo, being expressed in what looks like blood islands (Fig. $2 \mathrm{C}, \mathrm{F}$ and $\mathrm{G}$ ).

At $\mathrm{HH} 10$ a very weak staining is detected in the mesencephalon (Fig. 1A-blue arrowheads). By the end of HH12 MGP appears to be absent from the mesencephalon but present very weakly in the prosencephalon and rhombencephalon (Fig. 1B-blue arrowheads). In addition to this expression, two stripes bilateral to the mesencephalon can be observed, possibly of mesenchymal nature (Fig. 1B-blue arrows). By the end of $\mathrm{E} 2$, from $\mathrm{HH} 17$ to $\mathrm{HH} 19$, the roof plate of the hindbrain becomes positive for MGP transcripts
(Fig. 2A, B and D).

By $\mathrm{HH} 18, M G P$ expression becomes discernible in the heart (Fig. 2B). Particularly, MGP is noticeable in the endocardium (Fig. 3Ea-Ed, yellow arrow), atrio-ventricular canal (AVC; Fig. 3Ea and $\mathrm{Eb}$, yellow asterisk), and in some regions of the atrium (Fig. 3Ec, yellow hashtag), sinus venosus (Fig. 3Ed, yellow square) and trabeculae (Fig. 3Ea and Eb, yellow arrowhead). At HH25 (or E4.5 - E5) our results show MGP being expressed partially in the atria, sinus venosus and trabeculae (Fig. 3Ga-Gc", respectively yellow hashtag, yellow square and yellow arrowheads), but no longer in the AVC (Fig. 3Gc, yellow asterisk).

We were able to identify MGP expression in the nephric duct (Fig. 3Ba-Ca and la-Id, green arrows) between $\mathrm{HH} 18$ and $\mathrm{HH} 23$.

\section{Discussion}

This is the first report of the expression pattern by whole-mount in situ hybridization (WISH) of MGP during the early stages of development of the avian embryo. Based on its expression pattern, we can speculate on its putative functions during organogenesis. MGP expression can be observed at the time and place where the preliminary dorsal aorta develops a vascular appearance. 


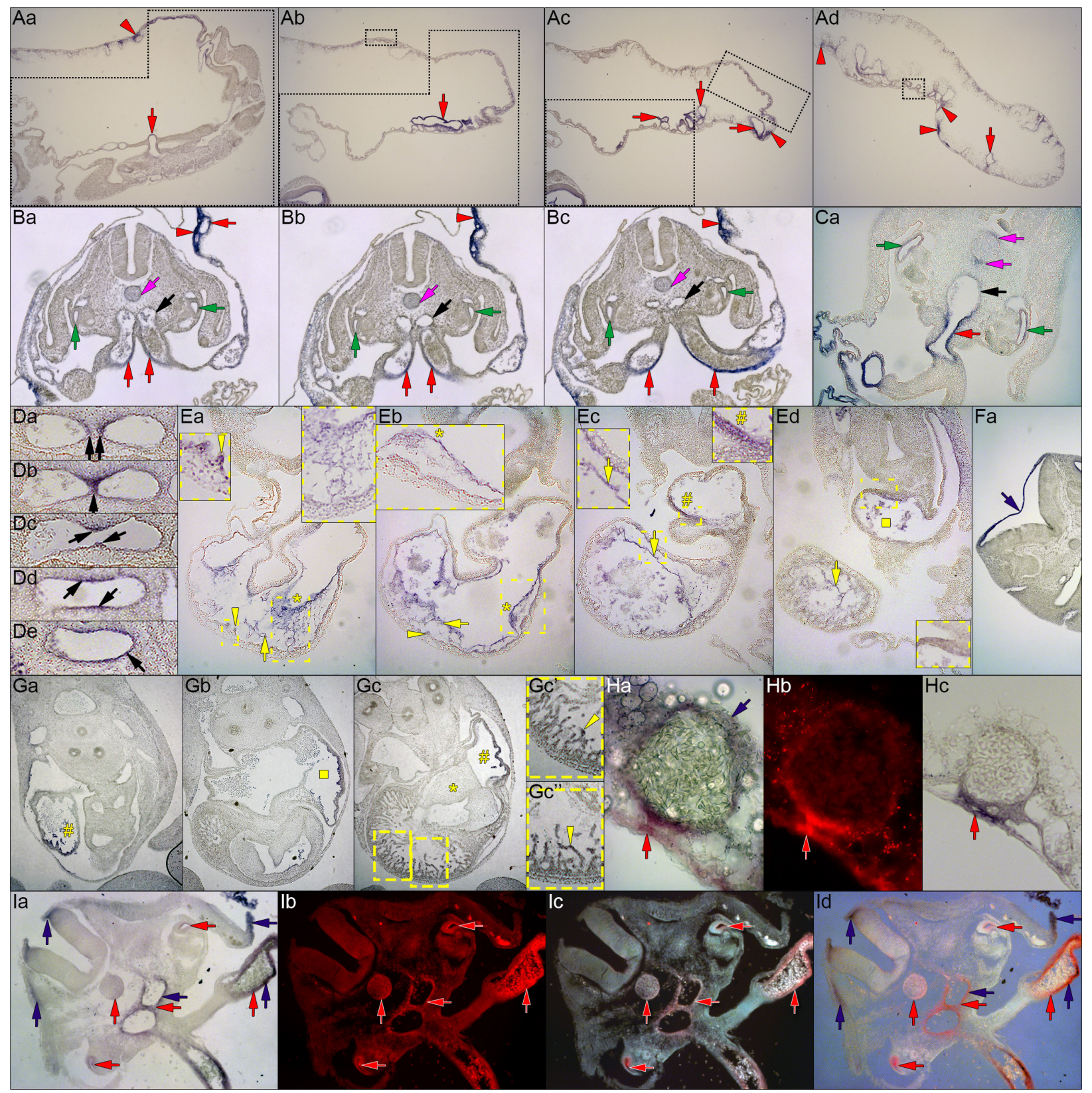

Fig. 3. MGP expression during yolk sac vasculature remodeling (histological sections). WISH using DIG labelled MGP (Aa-Gc"), and double WISH using DIG labelled MGP and fluorescein labelled Ephrin-B2 (Ha-ld). (A a-d) Longitudinal sections of an HH18 embryo (Figure 2B). Dashed lines delimitate the area pellucida. Red arrows, uniformly stained vessel; red arrowheads, dorsal part of vitelline artery branches and vicinity of several vessels and venules. (B a-c) Tranverse sections of an HH18 embryo. MGP expression is seen in the notochord (pink arrows), dorsal aorta (black arrows), nephric duct (green arrows), vitelline arteries and vessels, venueles (red arrows) and its vicinity in the area opaca (red arrowheads). (Ca) Tranverse sections of an HH23 embryo. MGP expression is seen in the vicinity of the notochord (pink arrows), dorsal aorta (black arrow), nephric duct (green arrows), and vitelline arteries (red arrow). (D a-e) Magnification of the carotids and dorsal aorta from an HH18 embryo (Figure 2 panel B). Black arrows show how MGP expression changes across the embryo, from the head (carotids) until beneath the heart (dorsal aorta). (E a-d) Transverse sections of an HH18 embryo (Figure 2B) at the heart level. MGP expression is observed in the endocardium (yellow arrow), atrio-ventricular canal (yellow asterisk), trabeculae (yellow arrowhead), in some regions of the atrium (yellow hastag) and in the sinus venosus (yellow square). Insets show higher magnifications of the areas within the yellow dashed boxes. (Fa) Transverse section of a roof plate (blue arrow) from an embryo at HH23. (G a-c) Transverse sections of an HH25 embryo at the heart level. MGP expression is observed partially in both atria (yellow hastag), sinus venosus (yellow square) and trabeculae (yellow dashed line box), but no longer in the AVC (yellow asterisk). (G c'-c") Magnification of yellow dashed line boxes in panel Gc showing MGP expression in the trabeculae (yellow arrowhead). (Ha) Double WISH section of a vessel in the area opaca, in bright field. HH18 embryo. Red arrow, MGP expression; blue arrow, Ephrin-B2 expresion. (Hb) Image in panel Ha using fluorescence. Red arrow, MGP expression. (Hc) Detail of a vessel in the area opaca positive for MGP (red arrow). WISH section from another embryo at HH18, in bright field. (I a-d) Double WISH transverse section of an embryo at HH18. In bright field (la), fluorescence (Ib, only red channel; Ic, red channel and empty channel), combined view of fluorescence and bright field view (Id). Red arrows, MGP expression; blue arrows, Ephrin -B2 expression. 
This process is known to take a few hours, and occurs at E1.5 or by $\mathrm{HH} 10$ (Sato 2013). The anterior ends of the dorsal aortae are connected with outflow tracts, and the posterior ends gradually elongate toward the tail, giving rise to the primary dorsal aortae that posteriorly connect to the vitelline arteries (also known as omphalo-mesenteric arteries; Sato 2013).

At E2, at the moment of the onset of circulation, MGP expression can still be seen on what appears to be the anterior part of the dorsal aorta. These MGP positive areas are situated along the first 7 somites (Fig. $1 A$ and $B$, black arrows). Although it seems likely that the presence of $M G P$ in these areas is related to dorsal aortae rearrangement, other hypotheses emerge. (1) MGP could be involved in the epithelial-mesenchymal transformations that occur by this developmental stage in the trunk of the chick embryo: the formation of neural crest from neural tube and formation of sclerotome mesenchyme from ventromedial somite wall. Other matrix proteins, such as type I collagen, have been proposed to play a role in directing neural crest migration. Interestingly, type I collagen and MGP are both skeletogenesis-related proteins. (2) MGP could be expressed in the splanchnic and intermediate mesoderm, in a similar fashion as the transcription factor capsulin, playing a role in the urinary system development.

Previous studies show that the right and left dorsal aortae begin to fuse together, at $\mathrm{HH} 15$ (or E2,25; Fig. 1C). This process seems to be allowed by an anteroposterior wave of Chordin mRNA attenuation in the notochord, prior to dorsal aorta fusion (Garriock et al., 2010). Interestingly, at this developmental point, MGP expression is seen in the notochord and not in the dorsal aorta (Fig. 1C and Fig. 1ii, respectively pink and black arrows).

MGP may have a significant role in the formation of the yolk sac angiogenesis since it is expressed from the beginning on a plexus of small caliber sized vessels that will give rise to the vitelline arteries. MGP expression in the area vasculosa not only appears in the dorsal part of vitelline artery branches but also in the vicinity of several vessels and venules, as well as in what seems to be small vessels beneath main arterial branches (Fig. $3 \mathrm{Hc}, \mathrm{Aa}$ and $\mathrm{Bb}$ ). At 30ss, le Noble and colleagues (2004) described the presence of "blood-filled spots" identified as being small arteriolar side-branches of the vitelline artery that were selectively disconnected and broken off the arterial tree. These small arteriolar side-branches make sprouts perpendicular and dorsal to arteries, and reconnect to the primary venous system, being reperfused. These "blood-filled spots" represent the origin of the first embryonic veins to run parallel and dorsal to an artery, i.e. the lateral vitelline veins, as well as of all the other veins of the secondary circulation. These findings may suggest that MGP could be somehow involved in this remodeling of arterial and venous trees, an hypothesis that is supported by the fact that spots of MGP expression are seen in several vessels in the yolk sac (Fig. 2B). Another hypothesis that seems reasonable to explain MGP expression in the vicinity of yolk sac vessels is the possibility that MGP plays a role in early definitive erythropoiesis. Hematopoietic and erythropoietic clusters in the yolk sac, residing outside the vasculature after the initiation of circulation at E2, have been suggested as a possible source for definitive erythrocytes (Sheng 2010). MGP was also observed on what appears to be another hematopoietic/erythropoietic cell population, the blood islands. Furthermore, it has been described that new blood islands are continuously being formed in the posterior part of the developing embryo, even at E3; and that these blood islands are molecularly distinguishable from those generated earlier (Sheng 2010).

MGP transcripts were also seen in the chick embryo's head at different moments in development. This fact is strengthened by a recent study, which reports that MGPgene deletion, a known BMP inhibitor, causes cerebral arteriovenous malformations (AVMs) in mice by activating activin receptor-like kinase 1 . The increase of this BMP type I receptor enhances the expression of Notch ligands Jagged 1 and 2, which increases Notch activity and alters the expression of Ephrin-B2 and Ephrin receptor B4, arterial and venous endothelial markers, respectively. MGP is responsible for maintaining the balance between BMP and Notch signaling and promotes a normal brain vasculature (Yao et al., 2013). Furthermore, earlier in 2013 another study highlighted MGP importance during angiogenesis, namely in promoting angiogenic resolution and vascular stabilization (Sharma and Albig 2013).

By the end of embryonic day 2, at $\mathrm{HH} 18$, MGP expression in the heart becomes discernible (Fig. 2B). Particularly, MGP is noticeable in the endocardium (Fig. 3Ea-Ed, yellow arrow), atrioventricular canal (AVC; Fig. 3Ea and Eb, yellow asterisk), and in some regions of the atrium (Fig. 3Ec, yellow hashtag), sinus venosus (Fig. 3Ed, yellow square) and trabeculae (Fig. 3Ea and Eb, yellow arrowhead. At HH25 (or E4.5 - E5) our results show $M G P$ being expressed partially in the atria, sinus venosus and trabeculae (Fig. 3Ga-Gc", respectively yellow hashtag, yellow square and yellow arrowheads), but no longer in the AVC (Fig. $3 \mathrm{Gc}$, yellow asterisk). During heart development, the AVC and the outflow tract region undergo an epithelial-mesenchymal transformation (EMT) to form the valves of the heart. These cushion areas are initially formed from cardiac jelly (ECM) which then becomes invaded by cells from the endocardium. The invasion is a result of molecular cues from the myocardium that signal the endothelium to activate. During activation, endothelial cells lose cell-cell contacts, undergo hypertrophy and polarization, and increase the expression of ECM molecules. In the chick embryo this invasion begins at the end of $\mathrm{HH} 16$ or beginning of $\mathrm{HH} 17$, after which activated endothelial cells become mesenchymal cells. Towards the end of E5, the AVC cushions meet and fuse in the midline, leaving a canal. Overall, this would indicate that MGP is being expressed by activated endothelial cells, being seen both in the endocardium and in the AVC. We can observe MGP also in the trabeculae, which are formed in an event where endocardial cells participate (DeLaughter et al., 2011). Interestingly, in addition to the fact that it is expressed in a layer of endocardium that covers the interior of the heart, it is known that Notch intracellular domain (NICD) is also detected in other endocardial populations such as the atrium and ventricles (DeLaughter et al., 2011). This expression pattern seems to correlate with MGP's, which could be modulating Notch pathway in those tissues.

During embryonic development, the intermediate mesoderm differentiates into tubular epithelial tissues of the kidney and genital system, and is first seen at $\mathrm{HH} 8+$ between the segmental plate and lateral plate (Hiruma and Nakamura 2003). By $\mathrm{HH} 9$ to $\mathrm{HH} 10$ it is a cell cord that goes from the level of the 6th to the presumptive 13th somite. This cell cord then separates into dorsal and ventral parts, becoming respectively the nephric duct and the tubules by HH14 (Hiruma and Nakamura 2003). Interestingly, the nephric duct is the outcome of one of the first mesenchymal-to-epithelial conversions (MET) to occur during development. The nephric 
duct induces surrounding intermediate mesoderm to differentiate into nephrons of the mesonephric kidney, the excretory organ of a majority of vertebrate species and a developmental intermediary excretory organ of birds and mammals. MGP expression in the nephric duct (Fig. 3 green arrows) is very interesting because: (1) it occurs in another epithelial tissue that endures a change in cellular nature; and (2) it is present in a structure that induces differentiation in its neighbor tissues.

Interestingly, MGP has been previously suggested to be associated in other biological systems, to endothelial-to-mesenchymal conversions (Yao et al., 2013), an hypothesis that is strengthened by the present results.

\section{Materials and Methods}

The studies involving animal experiments are in accordance to the ethical regulation for clinical research and European Union (EU) guidelines for animal research. All animal work performed in this study was conducted in compliance with the Portuguese law and approved by the Consultive Commission of the Veterinary Agency from Portuguese Ministry of Agriculture (Directive 2010/63/EU of the European Parliament), the Agency responsible for issuing approval for experimental animal studies, following the EU guidelines for animal research and welfare.

\section{Chick embryo collection}

Fertilized chicken eggs (Sociedade Agrícola Quinta da Freiria, SA, Torres Vedras, Portugal) were incubated for $1-4.5$ days at $38^{\circ} \mathrm{C}$ in a humidified incubator. Embryos were staged according to Hamburger and Hamilton.

\section{Whole-mount in situ hybridization}

MGP and EprinB2 antisense RNA (asRNA) probes were generated by incubating a DIG or FLUO-labeled ribonucleotide mix (Roche) with T3 or T7 RNA Polymerase (Roche) and the linearized DNA template, according to the manufacturer instructions. Template DNA used in the transcription reactions for asRNA production was generated by PCR using specific primers designed to obtain the complete coding sequence (CDS) for MGP, and a partial one for Ephrin-B2. Primer sequences are available upon request.

Embryos were processed for whole-mount in situ hybridization using a standard protocol as previously described (Furtado et al., 2014). MGP labeling was detected using BM purple alkaline phosfatase AP substrate. After in situ hibridization embryos were washed in PBS, dehydrated in a methanol series and paraffin embedded. Serial 8 - $12 \mu \mathrm{m}$ sections were taken (microtome Leica-RM 2135), dewaxed and rehydrated, mounted with DPX Mountant (Sigma-Aldrich), and analysed with Zeiss Axioimager Z2 microscope (Carl Zeiss Group). Double whole-mount in situ hybridization was performed for MGP and Ephrin-B2 and embryos processed for cryosectioning by embedding in tissue-tek ${ }^{\circledR}$ O.C.T. ${ }^{\mathrm{TM}}$ compound (Sakura). Serial $16 \mu \mathrm{m}$ sections were mounted with Mowiol Mountant Medium. MGP probe detection was performed by using the alkaline phosfatase (AP) substrate Texas Red (Roche) and visualized under fluorescent light; Ephrin-B2 was detected with BM Purple AP substrate and visualized in bright field. To identify unspecific auto-fluorescent signal green-channel images were obtained and juxtaposed. The imaging of the double-labeled criosections was performed with a Zeiss Axioimager Z2 microscope (Carl Zeiss Group) with a coupled Axiocam icc3 Digital Camera.

\section{Acknowledgements}

This work was supported by research grants from Fundação para a Ciência e Tecnologia (FCT), and the IBB/CBME, LA and CCMAR in the frame of project reference PEst-OE/EQB/LA0023/2011 and UID/
Multi/04326/2013 from FCT. NC is supported by a post-doctoral fellowship (SFRH/BPD/111898/2015) from FCT.

\section{References}

ALFIERI C M, CHEEK J, CHAKRABORTY S, YUTZEY K E (2010). Wnt signaling in heart valve development and osteogenic gene induction. Dev Biol 338: 127-135.

BOSTROM K, ZEBBOUDJ AF, YAO Y, LIN TS, TORRES A (2004). Matrix GLA protein stimulates VEGF expression through increased transforming growth factor-beta1 activity in endothelial cells. J Biol Chem279: 52904-52913.

CANCELA M L, LAIZÉ V, CONCEIÇÃO N (2014). Matrix Gla protein and osteocalcin: from gene duplication to neofunctionalization. Arch Biochem Biophys 1;561:56-63.

DELAUGHTER D M, SAINT-JEAN L, BALDWIN H S, BARNETT J V (2011). What chick and mouse models have taught us about the role of the endocardium in congenital heart disease. Birth Defects Res A Clin Mol Teratol 91: 511-255.

FURTADO J, BENTO M, CORREIA E, INÁCIO JM, BELO JA (2014). Expression and function of Ccbe1 in the chick early cardiogenic regions are required for correct heart development. PLoS One 29: e115481.

GARRIOCK R J, CZEISLER C, ISHII Y, NAVETTAA M, MIKAWA T (2010). An anteroposterior wave of vascular inhibitor downregulation signals aortae fusion along the embryonic midline axis. Development 137: 3697-3706.

HIRUMA T, NAKAMURA H (2003). Origin and development of the pronephros in the chick embryo. J Anat Dec 203: 539-552.

HUR DJ, RAYMOND G V, KAHLER S G, RIEGERT-JOHNSON D L, COHEN B A, BOYADJLEV S A (2005). A novel MGP mutation in a consanguineous family: review of the clinical and molecular characteristics of Keutel syndrome. Am J Med Genet A 135: 36-40.

KEUTEL J, JORGENSEN G, GABRIEL P (1971). [A new autosomal-recessive hereditary syndrome. Multiple peripheral pulmonary stenosis, brachytelephalangia, inner-ear deafness, ossification or calcification of cartilages]. Dtsch Med Wochenschr 96: 1676-1681.

LE NOBLE F, MOYON D, PARNANAUD L, YUAN L, DJONOV V, MATTHIJSEN R, BRÉANT C, FLEURY V, EICHMANN A (2004). Flow regulates arterial-venous differentiation in the chick embryo yolk sac. Development 131: 361-375.

LUO G, DUCYP, MCKEE MD, PINERO GJ, LOYERE, BEHRINGERRR, KARSENTLY $G$ (1997). Spontaneous calcification of arteries and cartilage in mice lacking matrix GLA protein. Nature 386: 78-81.

PRICE PA, CAPUTO JM, WILLIAMSON MK (2002). Bone origin of the serum complex of calcium, phosphate, fetuin, and matrix Gla protein: biochemical evidence for the cancellous bone-remodeling compartment. J Bone Miner Res 17:1171-1179.

SATO Y (2013). Dorsal aorta formation: separate origins, lateral-to-medial migration, and remodeling. Dev Growth Differ 55: 113-129.

SHARMA, B, ALBIG, A R (2013). Matrix Gla protein reinforces angiogenic resolution. Microvascular Res. 85: 24-33

SHENG G (2010). Primitive and definitive erythropoiesis in the yolk sac: a bird's eye view. Int J Dev Biol 54: 1033-1043.

WALLIN R, CAIN D, HUTSON S M, SANE D C, LOESER R (2000). Modulation of the binding of matrix Gla protein (MGP) to bone morphogenetic protein-2 (BMP2). Thromb Haemost 84:1039-1044.

WIEDEMANN M, TRUEB B, BELLUOCCIO D (1998). Molecular cloning of avian matrix Gla protein. Biochim Biophys Acta 1395: 47-49.

YAGAMIK, SUH JY, ENOMOTO-IWAMOTOM, KOYAMAE, ABRAMSWR, SHAPIRO IM, PACIFICI M, IWAMOTO M. (1999). Matrix GLA protein is a developmental regulator of chondrocyte mineralization and, when constitutively expressed, blocks endochondral and intramembranous ossification in the limb. J Cell Biol. 147:1097-1108.

YAO Y, JUMABAY M, WANG A, BOSTROM K I (2011). Matrix Gla protein deficiency causes arteriovenous malformations in mice. $J$ Clin Invest 121: 2993-3004.

YAOY, YAOJ, RADPARVAR M, BLAZQUEZ-MEDELAAM, GULHARD PJ, JUMABAY M, BOSTROM K I (2013). Reducing Jagged 1 and 2 levels prevents cerebral arteriovenous malformations in matrix Gla protein deficiency. Proc Natl Acad Sci. USA 110: 19071-19076. 


\section{Further Related Reading, published previously in the Int. J. Dev. Biol.}

Sox7 in vascular development: review, insights and potential mechanisms Jeanette J. Wat and Margaret J. Wat

Int. J. Dev. Biol. (2014) 58: 1-8

http://dx.doi.org/10.1387/ijdb.130323mw

The role of angiogenic growth factors in organogenesis

Enrico Crivellato

Int. J. Dev. Biol. (2011) 55: 365-375

http://dx.doi.org/10.1387/ijdb.103214ec

Signal transduction in vasculogenesis and developmental angiogenesis

Sunita Patel-Hett and Patricia A. D'Amore

Int. J. Dev. Biol. (2011) 55: 353-363

http://dx.doi.org/10.1387/ijdb.103213sp

Challenges and strategies for generating therapeutic patient-specific hemangioblasts and hematopoietic stem cells from human pluripotent stem cells

Ann Peters, Paul W. Burridge, Marina V. Pryzhkova, Michal A. Levine, Tea-Soon Park, Christopher Roxbury, Xuan Yuan, Bruno Péault and Elias T. Zambidis

Int. J. Dev. Biol. (2010) 54: 965-990

http://dx.doi.org/10.1387/ijdb.093043ap

The seminal work of Werner Risau in the study of the development of the vascular system Domenico Ribatti

Int. J. Dev. Biol. (2010) 54: 567-572

http://dx.doi.org/10.1387/ijdb.092892dr

5 yr ISI Impact Factor $(2013)=\mathbf{2 . 8 7 9}$
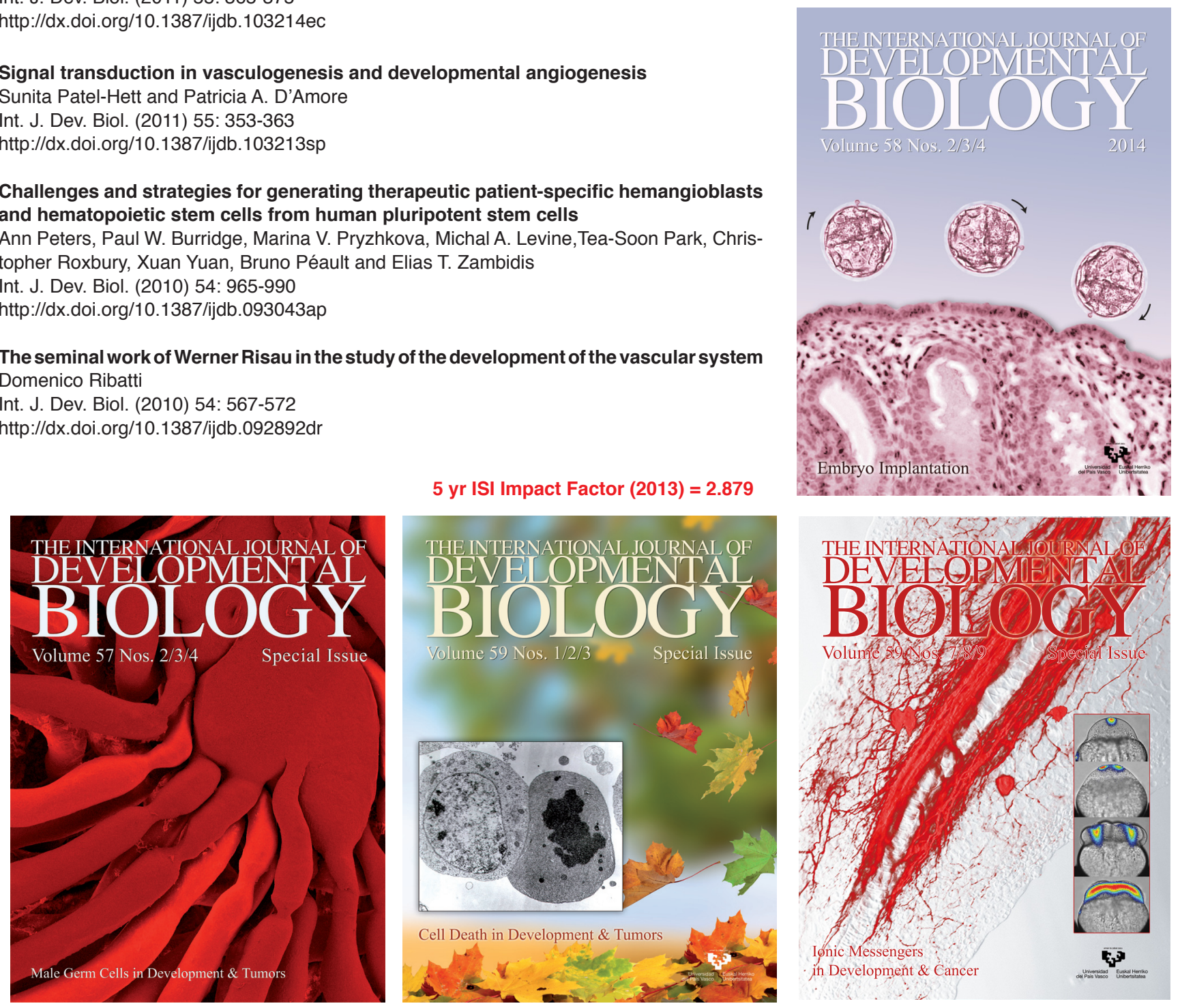